Article

\title{
HS-SPME Analysis of True Lavender (Lavandula angustifolia Mill.) Leaves Treated by Various Drying Methods
}

\author{
Jacek Łyczko $^{1, *(\mathbb{D}, \text { Klaudiusz Jałoszyński }}{ }^{2}$, Mariusz Surma ${ }^{2}$, Klaudia Masztalerz ${ }^{2}$ (D) \\ and Antoni Szumny ${ }^{1}$ (1) \\ 1 Faculty of Biotechnology and Food Science, Wrocław University of Environmental and Life Sciences, \\ Norwida 25, 50-375 Wrocław, Poland; antoni.szumny@upwr.edu.pl \\ 2 Institute of Agricultural Engineering, Wrocław University of Environmental and Life Sciences, \\ Chełmońskiego 37-41, 51-630 Wrocław, Poland; klaudiusz.jaloszynski@upwr.edu.pl (K.J.); \\ mariusz.surma@upwr.edu.pl (M.S.); klaudia.urbanska@upwr.edu.pl (K.M.) \\ * Correspondence: jacek.lyczko@upwr.edu.pl; Tel.: +48-71-320-51-47
}

Academic Editor: Constantinos K. Zacharis

Received: 31 January 2019; Accepted: 18 February 2019; Published: 20 February 2019

\begin{abstract}
True lavender (Lavandula angustifolia Mill.) is a widely used flavoring and medicinal plant, which strong aroma is mainly composed of linalool and linalyl acetate. The most valuable parts of the plant are the flowers, however leaves are also abundant in volatile constituents. One of the main factors responsible for its quality is the preservation procedure, which usually comes down to a drying process. For this reason an attempt to verify the influence of various drying methods (convective drying, vacuum-microwave drying and combined convection pre-drying with vacuum-microwave finishing drying) on the quality of true lavender leaves was carried out by determination of the volatile constituents profile by solid-phase microextraction (SPME) coupled with GC-MS technique. Total essential oil (EO) content was also verified. The study has revealed that the optimal drying method is strongly dependent on the purpose of the product. For flavoring properties convective drying at $60^{\circ} \mathrm{C}$ is the most optimal method, while the best for preserving the highest amount of EO is vacuum-microwave drying at $480 \mathrm{~W}$. Furthermore, SPME analysis had shown that drying may increase the value of true lavender leaves by significantly affecting the linalool to linalyl acetate to camphor ratio in the volatile profile.
\end{abstract}

Keywords: essential oil; drying; SPME; true lavender; volatile constituents

\section{Introduction}

Lavandula angustifolia Mill. (also named Lavandula officinalis Chaix)-the true lavender-is a essential oil-bearing plant known worldwide, which history of usage starts in Greek and Roman times and last up to this day. The entire genus belongs to the large Lamiacae family, which is mostly native to the Mediterranean region, however true lavender is a commonly growing plant in England, Europe, North America and Australia. The most valuable part of the plant are flowers due to their much higher essential oil content than leaves, and a favorable linalool to linalyl aceate to camphor ratio [1].

Nowadays due to the well-recognizable aroma lavender plants or their derivatives find applications in numerous ways, like in perfumery, cosmetics and household products, antimicrobial agents, food fragrance and flavor improvement or as food preservatives [1-3]. Furthermore, the essential oil obtained from lavender is an interesting object for trials considering biological activity and even in medicinal trials. Some studies and overviews from recent years mention the anti-aging, analgesic, nuroprotective, sedative or anticancer activities of lavender essential oil [2-9]. 
These various lavender essential oil applications are due to their unique chemical composition, rich in monoterpenes, sesquiterpenes, sesquiterpenoids, aliphatic compounds and especially an abundance of monoterpenoids [10], with linalool and linalyl acetate highlighted as main flower components [1,11,12]. In the case of the leaves the main essential oil constituents are eucalyptol (1,8-cineole), camphor and borneol [13-15].

As the main factors affecting the quality of the essential oils obtained from essential oils-bearing plants, plant chemotype, growing conditions and location, fertilizers used, time of harvesting and post-harvest treatment (including preservation method) are mentioned [2,11]. Among those factors, the preservation method has the most significant influence, where the most common one for plants rich in essential oils is drying [16-18]. Drying of essential oil-bearing plants allows one to obtain sustainable products with guaranteed quality, although it may cause also considerable losses of valuable constituents-mainly affecting the volatile constituents [17]. Furthermore, the color of the raw material may be strongly influenced by drying [16].

The traditional and natural method of drying uses solar radiation, however nowadays convective drying (CD), which uses flows of the hot air $[17,18]$, is the most common drying method used in natural products treatment. Nevertheless other techniques like freeze-drying, infrared drying, vacuum-microwave drying (VMD), spray drying or a combination of convective pre-drying with vacuum-microwave finishing drying (CPD-VMFD) are lately the objects of numerous investigations regarding natural products drying [18]. Unfortunately in case of drying the true lavender leaves only single factors were investigated. Interest in this topic is due to the necessity to find an optimal drying method for specific raw materials. In addition, not only a specific technique, but also its parameters, like drying time, temperature or pressure have a significant influence on the quality of the obtained products [19-22]. Overall the most important are air velocity and temperature-for plants the most suitable temperature is one between $50^{\circ} \mathrm{C}$ and $60^{\circ} \mathrm{C}$ [16].

The objective of this study was to determine the volatile profile composition and compound quantity of true lavender leaves and the influence of three drying methods (CD, VMD, CPD-VMFD) applied with various parameters. The study was done by a solid-phase microextraction (SPME) coupled with gas chromatography mass spectrometry technique (GC-MS). Also the total essential oil content was validated by using a hydrodistillation extraction technique.

\section{Results and Discussion}

\subsection{Drying Kinetics}

Figure 1 shows changes with time of the moisture ratio (MR) of leaf samples dehydrated by VMD at three magnetron powers (240,360 and $480 \mathrm{~W}$, Figure 1a), CD at temperatures in the range of 50 to $70{ }^{\circ} \mathrm{C}$ (Figure 1b), and combined (CPD-VMD) drying consisting of $\mathrm{CD}$ at $60^{\circ} \mathrm{C}$ and $\mathrm{VMD}$ at a magnetron power of $480 \mathrm{~W}$ (Figure 1c). The drying times, together with the maximum temperatures, the final moisture content and the constants of the Page model are listed in Table 1.

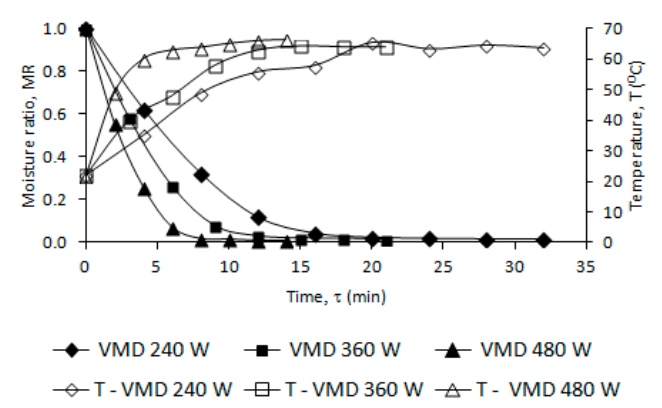

(a)

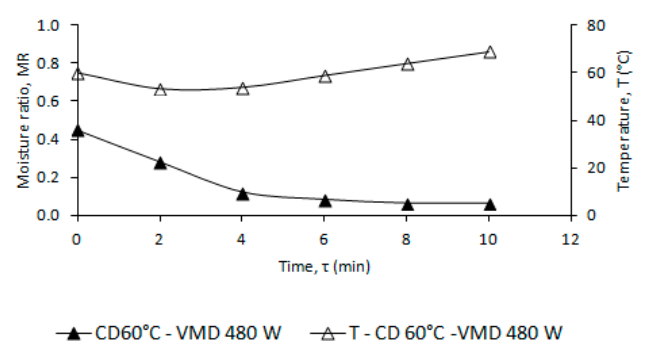

(b)

Figure 1. Cont. 


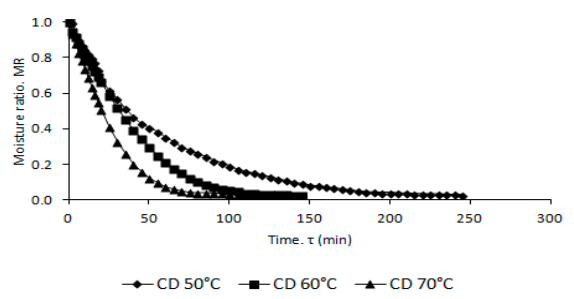

(c)

Figure 1. (a) Drying kinetics of true lavender leaves samples processed using VMD at magnetron powers 240, 360 and $480 \mathrm{~W}$; (b) Drying kinetics of true lavender leaves samples processed using CD at temperatures of 50,60 and $70{ }^{\circ} \mathrm{C}$; (c) Drying kinetics of true lavender leaves samples processed using VMFD at $480 \mathrm{~W}$ after $\mathrm{CPD}$ at temperature $60^{\circ} \mathrm{C}$.

Table 1. Final moisture content $\left(\mathrm{M}_{\mathrm{fwb}}\right)$, maximum temperature of the sample $\mathrm{T}$, convective drying time $(\tau)$, vacuum microwave drying time $\left(\tau_{1}\right)$, and constants $\mathrm{A}, \mathrm{k}$ and $\mathrm{n}$ of the modified Page model describing the drying kinetics.

\begin{tabular}{|c|c|c|c|c|c|c|c|c|c|}
\hline $\begin{array}{l}\text { Drying } \\
\text { Conditions }\end{array}$ & $A$ & $\begin{array}{c}\text { Constants } \\
K\end{array}$ & $n$ & $\mathbf{R}^{2}$ & RMSE & $\tau$ & $\tau_{1}$ & $\begin{array}{c}\mathrm{T} \\
\left({ }^{\circ} \mathrm{C}\right)\end{array}$ & $M_{f w b}(\%)$ \\
\hline $\mathrm{CD} 50^{\circ} \mathrm{C}$ & 1.000 & 0.0201 & 0.953 & 0.9984 & 0.0125 & 245 & - & 50 & 7.18 \\
\hline $\mathrm{CD} 60^{\circ} \mathrm{C}$ & 1.000 & 0.0125 & 1.173 & 0.9991 & 0.0104 & 145 & - & 60 & 7.09 \\
\hline $\mathrm{CD} 70^{\circ} \mathrm{C}$ & 1.000 & 0.0202 & 1.150 & 0.9983 & 0.0156 & 135 & - & 70 & 7.42 \\
\hline VMD $240 \mathrm{~W}$ & 1.000 & 0.0736 & 1.328 & 0.9989 & 0.0127 & - & 32 & 64 & 6.78 \\
\hline VMD $360 \mathrm{~W}$ & 1.000 & 0.1205 & 1.358 & 0.9991 & 0.0104 & - & 21 & 65 & 6.90 \\
\hline VMD $480 \mathrm{~W}$ & 1.000 & 0.2339 & 1.300 & 0.9991 & 0.0111 & - & 14 & 66 & 6.87 \\
\hline $\begin{array}{c}\text { CPD } 60^{\circ} \mathrm{C}+ \\
\text { VMFD } 480 \mathrm{~W}\end{array}$ & 0.449 & 0.2895 & 0.893 & 0.9982 & 0.0155 & 60 & 10 & 64 & 7.02 \\
\hline
\end{tabular}

The Page model can be successfully used to describe the drying kinetics of the true lavender leaves dehydrated by the CD, VMD and CPD-VMD methods, characterized by high values of the determination coefficient $\left(R^{2}>0.99\right)$ and low RMSE values $(<0.05)$. A good adaptation of the applied Page model for description of the drying kinetics can be found in many earlier publications of dill leaves, chanterelle and oyster mushrooms [23-25].

In the case of $\mathrm{CD}$ increasing the drying air temperature from 50 to $70{ }^{\circ} \mathrm{C}$ decreased the time of drying from 245 to $135 \mathrm{~min}$, respectively. In VMD drying, radical reductions in the total drying time have been observed: the time was shortened from 32 to $14 \mathrm{~min}$ with a power change from 240 to $480 \mathrm{~W}$. This radical reduction in the total drying time of VMD compared to CD is a result of the conventional water diffusion occurring, according to Fick's law, that is supported by a pressure diffusion mechanism of the Darcy type [26]. Combined CPD and VMFD using $480 \mathrm{~W}$, shortened the drying time of leaves almost 18 -fold compared to $\mathrm{CD}$ at $50{ }^{\circ} \mathrm{C}$. The use of $\mathrm{CD}$ and $480 \mathrm{~W}$ power caused a drop in the material temperature during VMD by $4{ }^{\circ} \mathrm{C}$ for leaves and $2{ }^{\circ} \mathrm{C}$ for flowers in reference to VMD 480 $\mathrm{W}$. This condition is caused by the molecular distribution of water particles inside the dried CD and the distribution of water particles has an effect on the generation of heat energy production under microwave radiation during VMD $[21,27,28]$. Energy consumption during the CD of plant materials is much lower than in VMD [29,30]. In industrial conditions, the best solution is a combined drying process consisting of $\mathrm{CPD}$ and VMFD. The $\mathrm{CD}$ is very effective at the beginning of the drying process (the largest loss of water occurs during that phase) and VMD at the final stage of drying (removal of water strongly bound to the cellular structure of the material being dried) $[18,27,28]$. The final choice of recommended drying process should be related to the aspects of the dried material (volatile composition and sensory attributes) [27,31]. 


\subsection{Volatile Constituents Profile of Fresh True Lavender Leaves}

HS-SPME analysis coupled with the GC-MS technique had revealed one hundred and four peaks (one as a two compound mixture) recognized as volatile constituents, of which only one hundred of them could be identified (the mass spectra of unidentified constituents are available in supplementary materials). Volatile constituents of true lavender leaves are listed in Table 2. Among them nineteen compounds were qualified as monoterpene hydrocarbons, twenty-six as oxygenated monoterpenes, twenty-four as sesquiterpene hydrocarbons, nine as oxygenated sesquiterpenes, ten as esters and eleven as others.

Table 2. Volatile constituents of fresh true lavender leaves.

\begin{tabular}{|c|c|c|c|c|c|}
\hline \multirow{2}{*}{ Compound } & \multirow{2}{*}{ RT (min) } & \multicolumn{3}{|c|}{ Retention Indeces (RI) } & \multirow{2}{*}{ Content $[\%]^{4}$} \\
\hline & & RI_lit ${ }^{1}$ & RI_lit ${ }^{2}$ & RI_exp ${ }^{3}$ & \\
\hline 1-Penten-3-ol & 2.407 & - & 684 & 686 & $\operatorname{Tr}^{5}$ \\
\hline (Z)-3-Hexenal & 3.755 & 797 & 810 & 808 & $0.23 \pm 0.14$ \\
\hline (E)-2-Hexenal & 4.765 & 846 & 854 & 857 & $0.33 \pm 0.17$ \\
\hline (Z)-3-Hexen-1-ol & 4.821 & 850 & 857 & 859 & $1.75 \pm 0.35$ \\
\hline 1-Hexanol & 5.087 & 863 & 868 & 871 & $0.32 \pm 0.09$ \\
\hline$(E, E)-2,4$-Hexadienal & 6.113 & 909 & 911 & 913 & $0.15 \pm 0.09$ \\
\hline 5.5-Dimethyl-1-vinylbicyclo[2.1.1]hexane & 6.380 & - & 921 & 924 & $\operatorname{tr}$ \\
\hline Tricyclene & 6.479 & 921 & 926 & 928 & $0.17 \pm 0.03$ \\
\hline$\alpha$-Thujene & 6.591 & 924 & 930 & 932 & $0.12 \pm 0.05$ \\
\hline$\alpha$-Pinene & 6.788 & 932 & 939 & 940 & $0.30 \pm 0.07$ \\
\hline Camphene & 7.209 & 946 & 954 & 955 & $0.92 \pm 0.19$ \\
\hline 3,7,7-Trimethyl-1.3.5-cycloheptatriene & 7.840 & - & 972 & 976 & $\operatorname{tr}$ \\
\hline Sabinene & 7.911 & 696 & 976 & 978 & $0.11 \pm 0.03$ \\
\hline 1-Octen-3-ol & 8.038 & 974 & 979 & 982 & $0.72 \pm 0.06$ \\
\hline 3-Octanone & 8.260 & 979 & 986 & 988 & $0.22 \pm 0.03$ \\
\hline$\beta$-Myrcene & 8.415 & 988 & 991 & 993 & $0.52 \pm 0.22$ \\
\hline Mesitylene & 8.512 & 994 & 995 & 996 & $\operatorname{tr}$ \\
\hline n-Decane & 8.681 & 1000 & 1000 & 1000 & $0.19 \pm 0.04$ \\
\hline$\alpha$-Phellandrene & 8.850 & 1002 & 1005 & 1007 & $0.49 \pm 0.28$ \\
\hline 3-Carene & 9.031 & 1008 & 1011 & 1013 & $1.60 \pm 0.66$ \\
\hline m-Cymene & 9.397 & 1020 & 1024 & 1026 & $2.58 \pm 0.33$ \\
\hline$p$-Cymene & 9.482 & 1022 & 1030 & 1028 & $4.81 \pm 0.52$ \\
\hline Limonene & 9.634 & 1024 & 1030 & 1033 & $3.42 \pm 1.16$ \\
\hline Eucalyptol & 9.692 & 1026 & 1031 & 1035 & $7.28 \pm 1.06$ \\
\hline$\beta$-cis-Ocimene & 9.902 & 1032 & 1038 & 1042 & $0.16 \pm 0.03$ \\
\hline$\beta$-trans-Ocimene & 10.240 & 1044 & 1050 & 1053 & $0.14 \pm 0.04$ \\
\hline$\gamma$-Terpinene & 10.605 & 1054 & 1059 & 1063 & $0.11 \pm 0.03$ \\
\hline trans-Sabinene hydrate & 10.886 & 1065 & 1070 & 1071 & $0.23 \pm 0.05$ \\
\hline cis-Linalool oxide & 11.041 & 1067 & 1074 & 1076 & $0.13 \pm 0.03$ \\
\hline unknown & 11.167 & - & - & 1079 & $\operatorname{tr}$ \\
\hline m-Cymenene & 11.419 & 1082 & 1085 & 1086 & $0.50 \pm 0.04$ \\
\hline$p$-Mentha-2.4(8)-diene & 11.519 & 1085 & 1088 & 1089 & $0.34 \pm 0.10$ \\
\hline$p$-Cymenene & 11.602 & 1089 & 1091 & 1091 & $0.30 \pm 0.03$ \\
\hline Camphenone & 11.840 & 1095 & 1096 & 1097 & $0.26 \pm 0.02$ \\
\hline Linalool & 11.953 & 1095 & 1096 & 1100 & $0.42 \pm 0.03$ \\
\hline 1.3.8-p-Menthatriene & 12.206 & 1108 & 1110 & 1108 & $0.10 \pm 0.02$ \\
\hline 1-Octen-3-ol acetate & 12.360 & 1110 & 1112 & 1114 & $3.80 \pm 0.52$ \\
\hline cis-p-Menth-2-en-1-ol & 12.556 & 1118 & 1121 & 1120 & $0.18 \pm 0.04$ \\
\hline trans-p-Mentha-2.8-dien-1-ol & 12.724 & 1119 & 1122 & 1125 & $0.64 \pm 0.19$ \\
\hline$c i s-p$-Mentha-2.8-dien-1-ol & 13.173 & 1133 & 1137 & 1139 & $0.26 \pm 0.03$ \\
\hline trans-p-Menth-2-en-1-ol & 13.327 & 1136 & 1140 & 1144 & $0.49 \pm 0.08$ \\
\hline Camphor & 13.496 & 1141 & 1146 & 1149 & $2.09 \pm 0.29$ \\
\hline Tetrahydrolavandulol & 13.960 & 1157 & 1161 & 1162 & $0.48 \pm 0.09$ \\
\hline Borneol + Lavandulol & 14.240 & 1165 & 1169 & 1170 & $4.66 \pm 0.69$ \\
\hline Melilotal & 14.450 & 1179 & 1182 & 1176 & $\operatorname{tr}$ \\
\hline
\end{tabular}


Table 2. Cont.

\begin{tabular}{|c|c|c|c|c|c|}
\hline \multirow{2}{*}{ Compound } & \multirow{2}{*}{ RT (min) } & \multicolumn{3}{|c|}{ Retention Indeces (RI) } & \multirow{2}{*}{ Content $[\%]^{4}$} \\
\hline & & RI_lit ${ }^{1}$ & RI_lit ${ }^{2}$ & RI_exp ${ }^{3}$ & \\
\hline Terpinen-4-ol & 14.631 & 1174 & 1177 & 1181 & $0.59 \pm 0.07$ \\
\hline m-Cymen-8-ol & 14.774 & 1176 & 1179 & 1184 & $2.09 \pm 0.25$ \\
\hline$p$-Cymen-8-ol & 14.914 & 1179 & 1182 & 1188 & $4.09 \pm 0.67$ \\
\hline$\alpha$-Terpineol & 15.082 & 1186 & 1189 & 1193 & $0.31 \pm 0.06$ \\
\hline Myrtenol & 15.278 & 1194 & 1195 & 1198 & $0.20 \pm 0.14$ \\
\hline trans-Piperitol & 15.671 & 1207 & 1208 & 1210 & $0.65 \pm 0.07$ \\
\hline cis-Carveol & 16.035 & 1215 & 1217 & 1222 & $0.37 \pm 0.07$ \\
\hline (Z)-Ocimenone & 16.159 & 1226 & 1229 & 1226 & $0.26 \pm 0.07$ \\
\hline exo-Fenchyl acetate & 16.356 & 1229 & 1232 & 1232 & $0.49 \pm 0.04$ \\
\hline cis-Verbenol & 16.623 & 1237 & 1244 & 1240 & $\operatorname{tr}$ \\
\hline Cumin aldehyde & 16.748 & 1238 & 1241 & 1244 & $1.92 \pm 0.59$ \\
\hline Carvone & 16.874 & 1246 & 1243 & 1247 & $1.08 \pm 0.28$ \\
\hline Geraniol & 17.055 & 1249 & 1252 & 1253 & $0.33 \pm 0.29$ \\
\hline Linalyl acetate & 17.263 & 1254 & 1257 & 1259 & $2.21 \pm 0.73$ \\
\hline Geranial & 17.529 & 1264 & 1267 & 1267 & $0.10 \pm 0.08$ \\
\hline trans-Carvone oxide & 18.021 & 1273 & 1276 & 1281 & $0.33 \pm 0.07$ \\
\hline Bornyl acetate & 18.301 & 1284 & 1285 & 1288 & $5.57 \pm 0.82$ \\
\hline Lavandulyl acetate & 18.428 & 1288 & 1290 & 1292 & $1.72 \pm 0.25$ \\
\hline Terpinen-4-ol acetate & 18.761 & 1299 & 1299 & 1301 & $0.18 \pm 0.02$ \\
\hline unknown & 19.124 & - & - & 1314 & $0.61 \pm 0.09$ \\
\hline Myrtenyl acetate & 19.435 & 1324 & 1326 & 1326 & $0.16 \pm 0.06$ \\
\hline$\delta$-Elemene & 19.749 & 1335 & 1337 & 1337 & $\operatorname{tr}$ \\
\hline$\alpha$-Terpinyl acetate & 20.036 & 1346 & 1349 & 1347 & $0.26 \pm 0.08$ \\
\hline$\alpha$-Cubebene & 20.179 & 1348 & 1351 & 1351 & $\operatorname{tr}$ \\
\hline$\alpha$-Longipinene & 20.351 & 1350 & 1352 & 1357 & $0.18 \pm 0.01$ \\
\hline unknown & 20.465 & - & - & 1361 & $0.31 \pm 0.05$ \\
\hline Silphiperfola-4.7(14)-diene & 20.578 & 1358 & 1362 & 1365 & $\operatorname{tr}$ \\
\hline Neryl acetate & 20.748 & 1359 & 1364 & 1371 & $0.26 \pm 0.06$ \\
\hline$\alpha$-Copaene & 21.134 & 1374 & 1376 & 1383 & $0.14 \pm 0.02$ \\
\hline Geranyl acetate & 21.248 & 1379 & 1381 & 1387 & $0.49 \pm 0.11$ \\
\hline$\alpha$-Bourbonene & 21.375 & 1387 & 1388 & 1391 & $\operatorname{tr}$ \\
\hline unknown & 21.461 & 1394 & 1396 & 1394 & $\operatorname{tr}$ \\
\hline$\beta$-Longipinene & 21.634 & 1400 & 1400 & 1399 & $0.26 \pm 0.06$ \\
\hline Sesquithujene & 21.833 & 1405 & 1405 & 1409 & $\operatorname{tr}$ \\
\hline$\alpha$-Cedrene & 22.049 & 1410 & 1411 & 1420 & $1.01 \pm 0.25$ \\
\hline (E)-Caryophyllene & 22.176 & 1417 & 1419 & 1427 & $6.11 \pm 1.48$ \\
\hline$\alpha$-Bergamotene & 22.506 & 1432 & 1435 & 1443 & $0.87 \pm 0.33$ \\
\hline Cadina-3.5-diene & 22.745 & - & 1458 & 1455 & $1.12 \pm 0.40$ \\
\hline$(E)$ - $\beta$-Farnesene & 22.889 & 1454 & 1457 & 1462 & $1.35 \pm 0.38$ \\
\hline cis-Muurola-4(15).5-diene & 23.084 & 1465 & 1466 & 1472 & $1.44 \pm 0.45$ \\
\hline 4-epi- $\alpha$-Acoradiene & 23.155 & 1474 & 1475 & 1475 & $0.19 \pm 0.00$ \\
\hline Germacrene D & 23.441 & 1484 & 1481 & 1489 & $0.58 \pm 0.17$ \\
\hline$\beta$-Himachalene & 23.629 & 1500 & 1500 & 1498 & $\operatorname{tr}$ \\
\hline unknown & 23.741 & 1502 & - & 1505 & $\operatorname{tr}$ \\
\hline$\alpha$-Bulnesene & 23.840 & 1509 & 1509 & 1511 & $0.92 \pm 0.16$ \\
\hline$\gamma$-Cadinene & 24.023 & 1513 & 1513 & 1523 & $10.53 \pm 1.51$ \\
\hline cis-Calamenene & 24.149 & 1528 & 1529 & 1531 & $0.65 \pm 0.07$ \\
\hline 10-epi-Cubebol & 24.290 & 1533 & 1535 & 1540 & $0.11 \pm 0.05$ \\
\hline$\alpha$-Cadinene & 24.402 & 1537 & 1538 & 1547 & $0.12 \pm 0.02$ \\
\hline Cadala-1(10).3.8-triene & 24.473 & - & 1555 & 1552 & $\operatorname{tr}$ \\
\hline trans-Cadinene ether & 24.669 & 1557 & - & 1564 & $0.35 \pm 0.09$ \\
\hline unknown & 24.851 & - & - & 1576 & $0.13 \pm 0.05$ \\
\hline Spathulenol & 24.950 & 1577 & 1578 & 1582 & $0.25 \pm 0.05$ \\
\hline Caryophyllene oxide & 25.158 & 1582 & 1583 & 1595 & $3.31 \pm 0.18$ \\
\hline 1-epi-Cubenol & 25.552 & 1627 & 1628 & 1628 & $0.56 \pm 0.03$ \\
\hline$\tau$-Cadinol & 25.860 & 1635 & 1340 & 1656 & $2.04 \pm 0.55$ \\
\hline unknown & 26.056 & - & - & 1673 & $0.11 \pm 0.03$ \\
\hline 14-Hydroxy-4.5-dihydrocaryophyllene & 26.407 & 1706 & 1706 & 1706 & $0.21 \pm 0.11$ \\
\hline unknown & 26.911 & 1760 & 1761 & 1764 & $0.23 \pm 0.02$ \\
\hline
\end{tabular}

${ }^{1}$ Retention indices according to Adams [32]; ${ }^{2}$ Retention indices according to NIST14 database; ${ }^{3}$ Relative retention indices calculated against $n$-alkanes; ${ }^{4} \%$ calculated from TIC data; ${ }^{5}$ tr. $<0.1 \%$. 
The main headspace volatile constituents of the examined true lavender leaves samples were $p$-cymen-8-ol $(4.09 \% \pm 0.67)$, a mixture of borneol and lavandulol $(4.66 \% \pm 0.69)$, o-cymene $(4.81 \% \pm 0.52)$, bornyl acetate $(5.57 \% \pm 0.82),(E)$-caryophyllene $(6.11 \% \pm 1.48)$, eucalyptol $(7.28 \%$ $\pm 1.06)$ and $\gamma$-cadinene $(10.53 \pm 1.51)$. In less amounts cumin aldehyde $(1.92 \% \pm 0.59), \tau$-cadinol $(2.04 \% \pm 0.55), m$-cymen-8-ol $(2.09 \% \pm 0.25)$, camphor $(2.09 \% \pm 0.92), p$-cymene $(2.58 \% \pm 0.33)$, caryophyllene oxide $(3.31 \% \pm 0.18)$, limonene $(3.42 \% \pm 1.16)$ and 1 -octen-3-pl acetate $(3.80 \% \pm 0.52)$, which have a significant influence on true lavender leaves' fragrance quality, were identified. The most characteristic and valuable constituents for true lavender (flowers), linalool and linalyl acetate, represented $0.42 \% \pm 0.03$ and $2.21 \% \pm 0.73$ of the total amount of volatile constituents, respectively.

Similar findings were reported in recent studies where eucalyptol $(8.50 \%$ and $31.9 \%)$, borneol (15.21\% and $24 \%)$, camphor $(2.00 \%$ and $16.1 \%)$, cumin aldehyde $(0.50 \%$ and $2.2 \%)$ were identified as main volatile components of a true lavender leaves sample [33,34]. Also, one of these studies, by Hassanpouraghdam et al. [34] pointed out low amounts or even a lack of linalool (0.7\%) and linalyl acetate. This result is contrary to the one obtained in this study, however it may be related to the slightly different plant chemotype or due to the fact that in Hassanpouraghdam's study leaves essential oil was analyzed, not headspace volatiles. Nurzyńska-Wierdak and Zawiślak [35] have identified linalool and linalyl acetate in a similar ratio (1:5), and furthermore they also found higher amounts of $\gamma$-cadinene (3.4 \pm 0.1$)$ and caryophyllene oxide $(7.2 \% \pm 0.2)$.

Unfortunately, there is a lack of reports in literature including HS-SPME analysis of true lavender leaves volatile constituents. Most of available ones takes as study object lavender flowers or whole aerial parts of the plant, where linalool and linalyl acetate dominate in the chromatographic profile of the volatile constituents [36-38]. Torabbeigi and Aberoomand Azar [39] reported high amounts of eucalyptol (41.37\%), camphor (15.83\%), borneol (12.32\%), $\alpha$-pinene $(4.66 \%)$, and $\gamma$-cadinene $(1.07 \%)$ found by HS-SPME analysis of true lavender samples. At the same time they did not find any traces of linalool or linalyl acetate, suggesting that the major part of their samples were lavender leaves.

\subsection{Effect of the Drying Methods on the Quantity of True Lavender Leaves Volatile Constituents}

In the fresh true lavender leaves cultivated in Poland used in this study the content of essential oil was $3.082 \mathrm{~g}$ per $100 \mathrm{~g}^{-1}$ of DW. Overall this essential oil yield is high in comparison to previously reported ones, as Mirahmadi and Norouzi [40] obtained just 2.34\% of essential oil from true lavender. Moreover, Milojević et al. [41] report the essential oil yield in sage and eucalyptus leaves ranges from $2 \%$ up to $2.87 \%$. Changes of essential oil content, the concentration of sixteen major volatile constituents and linalool caused by the various drying methods are shown in Table 3.

In the case of essential oil content all applied drying methods significantly affected the raw material. The most efficient method was VMD $480 \mathrm{~W}\left(1.302 \mathrm{~g}\right.$ per $\left.100 \mathrm{~g}^{-1}\right)$, followed by VMD $240 \mathrm{~W}$


overlapping significant groups. The percent recovery of essential oil in these methods were as follows $42.26 \%, 34.87 \%, 32.19 \%$ and $29.87 \%$, in comparison to the amount of essential oil obtained from fresh sample. The less efficient drying method was $\mathrm{CD} 50^{\circ} \mathrm{C}$, with a $19.06 \%$ recovery. The ratios of percent recovery between fresh sample and ones subjected to drying are presented in Figure 2. Baydar and Erbaş [42], Figiel et al. [19], Ghasemi et al. [43] found as well that due to the applied drying method or its parameters the decrease in essential oil yield of green plant parts may range as high as three to five times. Furthermore, Politowicz et al. [24] and Nöfer et al. [27], in the case of mushroom drying, observed similar effects to the ones found in this study. 
Table 3. Variability of major volatile constituents, linalool, and total essential oil of true lavender leaves caused by various drying methods.

\begin{tabular}{|c|c|c|c|c|c|c|c|c|}
\hline \multirow{3}{*}{ Compound } & \multicolumn{8}{|c|}{ Drying Method } \\
\hline & Fresh & $\mathrm{CD} 50^{\circ} \mathrm{C}$ & $\mathrm{CD} 60^{\circ} \mathrm{C}$ & $\mathrm{CD} 70^{\circ} \mathrm{C}$ & CPD-VMFD & VMD $240 \mathrm{~W}$ & VMD $360 \mathrm{~W}$ & VMD $480 \mathrm{~W}$ \\
\hline & \multicolumn{8}{|c|}{ Content $[\%]^{1}$} \\
\hline$p$-Cymene & $2.58^{\mathrm{a}}$ & $2.73^{c}$ & $2.72^{c}$ & $1.76^{\mathrm{d}}$ & $2.01^{\mathrm{d}, \mathrm{e}}$ & $2.15^{\mathrm{e}}$ & $2.27^{\mathrm{e}}$ & $3.50^{b}$ \\
\hline$o$-Cymene & $4.81^{\mathrm{a}}$ & $6.26^{c}$ & $5.65^{d}$ & $3.05^{\mathrm{f}}$ & $3.69^{\mathrm{e}}$ & $4.62^{\mathrm{g}}$ & $4.73^{\mathrm{g}}$ & $8.08^{b}$ \\
\hline Limonene & $3.42^{\mathrm{a}}$ & $3.27^{\mathrm{f}}$ & $3.47^{\mathrm{f}}$ & $1.31^{\mathrm{e}}$ & $3.08^{c, f}$ & $1.97^{\mathrm{d}, \mathrm{e}}$ & $2.41^{\mathrm{c}, \mathrm{d}}$ & $6.99^{b}$ \\
\hline Eucalyptol & $7.28^{\mathrm{a}}$ & $5.01^{b, c}$ & $3.71^{\mathrm{d}}$ & $5.12^{b}$ & $3.98^{c, d}$ & $3.25^{\mathrm{d}}$ & $3.74^{\mathrm{d}}$ & $3.44^{\mathrm{d}}$ \\
\hline 1-Octen-3-ol. acetate & $3.80^{\mathrm{a}}$ & $2.70^{\mathrm{d}, \mathrm{e}}$ & $2.82^{\mathrm{d}, \mathrm{e}}$ & $4.23^{c}$ & $6.22^{b}$ & $2.10^{\mathrm{e}}$ & $4.42^{c}$ & $3.68^{\mathrm{c}, \mathrm{d}}$ \\
\hline Camphor & $2.09^{a}$ & $2.32^{b}$ & $1.40^{\mathrm{d}}$ & $1.89^{\mathrm{c}}$ & $0.40^{\mathrm{f}}$ & $1.12^{\mathrm{e}}$ & $0.34^{\mathrm{f}}$ & $0.39^{f}$ \\
\hline Borneol + Lavandulol & $4.66^{\mathrm{a}}$ & $7.63^{b}$ & $5.75^{\mathrm{d}}$ & $6.07^{\mathrm{d}}$ & $1.37^{\mathrm{e}}$ & $4.78^{\mathrm{c}}$ & $1.46^{\mathrm{e}}$ & $1.35^{\mathrm{e}}$ \\
\hline$m$-Cymen-8-ol & $2.09^{\mathrm{a}}$ & $3.18^{\mathrm{c}}$ & $2.48^{\mathrm{d}}$ & $3.67^{b}$ & $0.02 \mathrm{e}$ & $2.74^{\mathrm{d}}$ & $0.08^{\mathrm{e}}$ & $0.07^{\mathrm{e}}$ \\
\hline$p$-Cymen-8-ol & $4.09^{\mathrm{a}}$ & $6.31^{\mathrm{c}}$ & $6.05^{c}$ & $7.17^{b}$ & $1.10^{\mathrm{d}}$ & $6.07^{c}$ & $1.04^{\mathrm{d}}$ & $0.89^{\mathrm{d}}$ \\
\hline Cumin aldehyde & $1.92^{\mathrm{a}}$ & $3.59^{\mathrm{c}}$ & $3.74^{\mathrm{c}}$ & $4.48^{\mathrm{b}}$ & $0.59^{\mathrm{d}}$ & $4.30^{b}$ & $0.66^{\mathrm{d}}$ & $0.59^{\mathrm{d}}$ \\
\hline Linalyl acetate & $2.21^{\mathrm{a}}$ & $3.46^{\mathrm{d}}$ & $11.06^{\mathrm{b}}$ & $4.23^{\mathrm{d}}$ & $1.60^{\mathrm{e}}$ & $5.29^{c}$ & $1.66^{\mathrm{e}}$ & $1.75^{\mathrm{e}}$ \\
\hline Bornyl acetate & $5.57^{\mathrm{a}}$ & $3.54^{\mathrm{c}}$ & $2.36^{\mathrm{e}}$ & $4.04^{b}$ & $0.07^{\mathrm{f}}$ & $3.07^{\mathrm{d}}$ & $0.07^{\mathrm{f}}$ & $0.14^{\mathrm{f}}$ \\
\hline Caryophyllene $<(\mathrm{E})->$ & $6.11^{\mathrm{a}}$ & $2.11^{\mathrm{d}}$ & $2.78^{c}$ & $3.51^{\mathrm{f}}$ & $6.38^{b}$ & $4.85^{\mathrm{e}}$ & $5.28^{\mathrm{e}}$ & $3.98^{f}$ \\
\hline$\gamma$-Cadinene & $10.53^{\mathrm{a}}$ & $3.67^{\mathrm{f}}$ & $4.43^{\text {ef }}$ & $4.74^{\mathrm{e}}$ & $8.48^{c, d}$ & $7.80^{\mathrm{d}}$ & $9.20^{\mathrm{c}}$ & $5.88^{b}$ \\
\hline Caryophyllene oxide & $3.31^{\mathrm{a}}$ & $2.43^{c}$ & $1.63^{b}$ & $2.12^{b, c}$ & $2.24^{b, c}$ & $2.11^{b, c}$ & $2.47^{\mathrm{c}}$ & $1.89^{b, c}$ \\
\hline$\tau$-Cadinol & $2.04^{\mathrm{a}}$ & $1.62^{d}$ & $1.44^{\mathrm{d}}$ & $2.78^{c}$ & $2.74^{\mathrm{c}}$ & $3.37^{b, c}$ & $3.85^{b}$ & $1.74^{\mathrm{d}}$ \\
\hline$\Sigma$ & $66.51^{\mathrm{a}}$ & $59.83^{b}$ & $61.42^{b}$ & $60.17^{b}$ & $43.97^{c}$ & $59.59^{b}$ & $43.68^{c}$ & $42.47^{c}$ \\
\hline Linalool & $0.38^{\mathrm{a}}$ & $4.32^{\mathrm{c}}$ & $6.33^{b}$ & $4.71^{\mathrm{c}}$ & $0.76^{\mathrm{a}, \mathrm{d}}$ & $6.62^{b}$ & $0.73^{\mathrm{a}, \mathrm{d}}$ & $1.16^{\mathrm{d}}$ \\
\hline TOTAL essential oil $\left[\mathrm{mL} 100 \mathrm{~g}^{-1} \mathrm{dw}\right]^{2}$ & $3.082^{\mathrm{a}}$ & $0.588^{\mathrm{e}}$ & $0.726^{\mathrm{f}}$ & $0.992^{\mathrm{c}, \mathrm{d}}$ & $0.921^{b, c}$ & $1.075^{\mathrm{d}}$ & $0.881^{\mathrm{b}}$ & $1.302^{\mathrm{g}}$ \\
\hline
\end{tabular}

${ }^{1}$ Values followed by the same letter within a row are not significantly different $\left(p>0.05\right.$, Duncan's test); ${ }^{2}$ Values obtained from steam distillation in Deryng apparatus. 


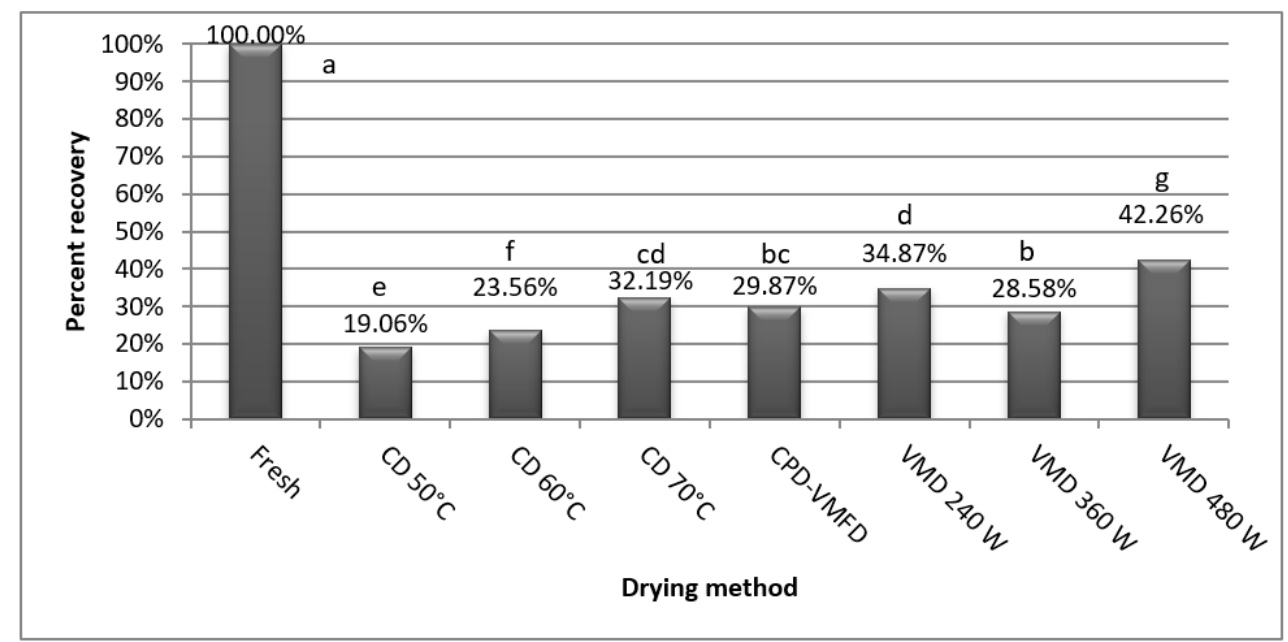

Figure 2. Percent recovery of essential oil of true lavender leaves after applying various drying methods.

The total essential oil content results are not equivalent to the content of sixteen major constituents. In fresh true lavender leaves, sixteen major constituents accounting for $66.51 \%$ of the total volatile constituents and changes caused by all drying methods were significantly distinct. Less differences were observed in the CD and CPD-VMFD methods (5.09-6.68 percentage points) and the highest were observed for the VMD method (22.54-24.04 percentage points). Further, some results in the case of particular constituents among the sixteen major ones are worth underlining. Again, all drying methods had a significant influence on a particular constituent share of total volatile constituents. The most interesting was the increase a share of linalyl acetate (even up to $11.06 \%$ of the share in the $\mathrm{CD} 60^{\circ} \mathrm{C}$ method) along with the decrease of camphor share (down to $1.40 \%$ ) at the same time. Also the share of linalool, the main aroma compound for true lavender, increased significantly after all drying treatments, except for VMD $240 \mathrm{~W}$ and VMD $360 \mathrm{~W}$. These results suggest that applying drying, mainly CD, for true lavender leaves, may improve the characteristics for use in flavoring, in accordance with Kim and Lee [44] and Da Porto and Decorti [45], who report that the high ratio of linalool and linalyl acetate to camphor ratio is an important quality marker for lavender fragrance. Similar changes after applying drying were obtained by Śmigielski et al. [10]. Nevertheless, if the aim is to preserve as much essential oil as possible, the VMD methods would be more applicable. Very poor results, both in case of total essential oil and major volatile constituents, were obtained after the CPD-VMFD method, what is in contradiction with results obtained by Szumny et al. [20] for rosemary drying (R. officinalis), however the taxonomic differences between rosemary and true lavender should be considered.

\section{Materials and Methods}

\subsection{Plant Material}

The drying process was carried out on true lavender cultivated in Poland (Kawon-Hurt Nowak Sp.j. Company, Gostyń, Poland). The initial moisture content of material was $2.7 \mathrm{~kg}$ per $\mathrm{kg}$ of dry weight. The drying processes were stopped after no further change in weights was observed. Moisture content of samples was determined using a vacuum dryer (SPT-200. ZEAMIL Horyzont, Krakow, Poland).

\subsection{Drying Methods}

\subsubsection{Convective Drying (CD)}

$\mathrm{CD}$ was performed using the equipment designed and constructed at the Institute of Agricultural Engineering (Wrocław University of Environmental and Life Sciences, Wrocław, Poland). Samples 
were placed in the container $(\mathrm{d}=100 \mathrm{~mm})$ and dried at $50{ }^{\circ} \mathrm{C} .60^{\circ} \mathrm{C}$ and $70{ }^{\circ} \mathrm{C}$-all with an air velocity of $0.5 \mathrm{~ms}^{-1}$.

\subsubsection{Vacuum-Microwave Drying (VMD)}

VMD was performed on samples with a SM 200 dryer (Plazmatronika, Wrocław, Poland). The dryer was equipped in cylindrical drum made of glass $(18 \mathrm{~cm}$ of diameter $\times 27 \mathrm{~cm}$ of length). The drum with glass rotated with $6 \mathrm{rev} \cdot \mathrm{min}^{-1}$. In the dryer system there was a BL 30P vacuum pump (Tepro, Koszalin, Poland), an MP 211 vacuum gauge (Elvac, Bobolice, Poland) and a compensation reservoir of $0.15 \mathrm{~m}^{3}$ capacity and a cylindrical tank. In this study, three power levels $(240,360$ and $480 \mathrm{~W}$ ) and pressures ranging from of 4 to $6 \mathrm{kPa}$ were used. The maximum temperature of dried lavender leaves was measured right after the removal from the dryer using an i50 infrared camera (Flir Systems AB, Stockholm, Sweden).

3.2.3. Combined Drying-Pre-Drying by Convective Drying with Vacuum-Microwave Finishing-Drying (CPD-VMFD)

CPD-VMFD performed on samples consisted of CPD at a temperature of $60{ }^{\circ} \mathrm{C}$ until a moisture content of leaves was around $0.45 \mathrm{~kg} \cdot \mathrm{kg}^{-1} \mathrm{db}$, was reached, followed by VMFD at $480 \mathrm{~W}$.

\subsection{Modeling of Drying Kinetics}

The drying kinetics of CD, VMD and CPD-VMFD were fitted based on the mass losses of the true lavender samples. For CD, weight losses were monitored every $2 \mathrm{~min}$ for the initial $20 \mathrm{~min}$ and then every 5 min thereafter until the end of the drying process.

VMD samples were monitored every 2, 3 and 4 min for 480, 360 and $240 \mathrm{~W}$. Different drying time intervals were applied in order to ensure a similar energy input between subsequent measurements regardless of the microwave power level.

The moisture ratio (MR) of lavender leaves during drying experiments was calculated using the following equation:

$$
M R=\frac{M_{(t)}-M_{e}}{M_{o}-M_{e}}
$$

where $\mathrm{M}_{(\mathrm{t})}$ is the moisture content at time $\tau . \mathrm{M}_{\mathrm{o}}$ is the initial moisture content, and $\mathrm{M}_{\mathrm{e}}$ is the equilibrium moisture content ( $\mathrm{kg}$ water $/ \mathrm{kg}$ dry matter). The values of $\mathrm{M}_{\mathrm{e}}$ are relatively small comparing to those of $\mathrm{M}_{(\mathrm{t})}$ or $\mathrm{M}_{\mathrm{o}}$. The error due to the simplification is negligible [46-48], thus the moisture ratio was calculated as follows:

$$
M R=\frac{M_{(t)}}{M_{0}}
$$

Table Curve 2D Windows v2.03 was used to fit the basic drying models to the measured MR determined accordingly to Equation (2). There are several drying models in the literature that can be used to describe the kinetics of drying plant materials. For drying model selection, drying curves were fitted to five well known thin drying models, including the modified Page model. Henderson-Pabis, logarithmic, Midilli-Kucuk, and Weibull ones. The best fit was determined using two parameters: the value of the coefficient of determination $\left(\mathrm{R}^{2}\right)$ and root-mean squared error (RMSE). A model fits better if the value of $R^{2}$ is closer to 1 , and the RMSE value is closer to 0 , using the following equations:

$$
\begin{array}{r}
R^{2}=\frac{\sum_{i=1}^{N}\left(M R_{\text {pre }_{i}}-\overline{M R}_{\exp }\right)}{\sum_{i=1}^{N}\left(M R_{\exp _{i}}-\overline{M R}_{\exp }\right)} \\
R M S E=\sqrt{\frac{1}{N} \cdot \sum_{i=1}^{N}\left(M R_{\exp _{i}}-M R_{\text {pre }_{i}}\right)^{2}}
\end{array}
$$


where $M R$ is moisture ratio, $\overline{M R}$ is the mean value of moisture ratio, "pre" and "exp" indicate predicted and experimental values, respectively, while " $i$ " indicates subsequent experimental data and $N$ is the number of observations.

Tests conducted in this study proved that the best fitting was obtained for the modified Page model:

$$
M R=A \exp \left(-k \tau^{n}\right)
$$

where $A, n$, and $k$ are constants.

\subsection{Solid-Phase Micro Extraction (SPME) Analysis}

HS-SPME analysis (30 min exposure to a $2 \mathrm{~cm}$ DVB/CAR/PDMS fiber, Supelco, Bellefonte, PA, USA, followed by analyte desorption at $220^{\circ} \mathrm{C}$ for $3 \mathrm{~min}$ ) was performed on Varian CP-3800/Saturn 2000 apparatus (Varian, Walnut Creek, CA, USA) equipped with a Zebron ZB-5 MSI ( $30 \mathrm{~m} \times 0.25 \mathrm{~mm}$ $\times 0.25 \mu \mathrm{m}$ ) column (Phenomenex, Shim-Pol, Poland). About $0.100 \mathrm{~g}$ of fresh or $0.150 \mathrm{~g}$ of dried sample was put in to headspace vials and kept in laboratory water bath at $70{ }^{\circ} \mathrm{C} .0 .5 \mathrm{mg}$ of 2-undecanone (Sigma Aldrich, Saint Louis, MO, USA) as an internal standard was added.

\subsection{GC-MS Analysis}

The GC oven temperature was programmed from $50{ }^{\circ} \mathrm{C}$, to $130{ }^{\circ} \mathrm{C}$ at rate $4.0^{\circ} \mathrm{C}$. then to $180^{\circ} \mathrm{C}$ at rate $10.0^{\circ} \mathrm{C}$, then to $280^{\circ} \mathrm{C}$ at rate $20.0^{\circ} \mathrm{C}$. Scanning was performed from 35 to $550 \mathrm{~m} / z$ in electronic impact (EI) mode at $70 \mathrm{eV}$. Samples were injected at a 1:10 split ratio and helium gas was used as the carrier gas at a flow rate of $1.0 \mathrm{~mL} \cdot \mathrm{min}^{-1}$. Analyses were run in triplicate.

\subsection{Hydrodistillation of Essential Oil (EO)}

Hydrodistillation of EOs was carried out by applying a Deryng apparatus. About $200 \mathrm{~g}$ of fresh sample or $100 \mathrm{~g}$ of dried sample was placed in $2 \mathrm{~L}$ round bottom flask with $500 \mathrm{~mL}$ of added distilled water. Yield was assessed as a measured volume of essential oil.

\subsection{Identification and Quantification of Volatile Compounds}

Identification of all volatile constituents obtained by HS-SPME analysis and hydrodistillation were based on comparison of experimentally obtained compound mass spectra with mass spectra available in NIST14 database. Also the experimentally obtained retention indeces (RI) by Kovats were compared with RI available in the NIST WebBook and literature data [32]. The quantification analysis was performed using ACD/Spectrus Processor (Advanced Chemistry Development, Inc., Toronto, $\mathrm{ON}$, Canada) through the integration of the peak area of the chromatograms.

\subsection{Statistical Analysis}

The data from drying kinetics were subjected to the analysis of variance using Tukey's test $(p<0.05)$ and the data from quantitative essential oil and volatile constituents were subjected to the analysis of variance using Duncan's test $(p<0.05)$, all using the STATISTICA 13.3 software for Windows (StatSoft, KrakowPoland).

\section{Conclusions}

One hundred constituents were identified in the volatile profile of true lavender leaves, with $p$-cymen-8-ol $(4.09 \% \pm 0.67)$, a mixture of borneol and lavndulol $(4.66 \% \pm 0.69), o$-cymene $(4.81 \%$ $\pm 0.52)$, bornyl acetate $(5.57 \% \pm 0.82)$, (E)-caryophyllene $(6.11 \% \pm 1.48)$, eucalyptol $(7.28 \% \pm 1.06)$ and $\gamma$-cadinene $(10.53 \pm 1.51)$ as a major ones. When various methods are applied during the drying process, this profile is strongly affected. The optimal drying method is dependent on the purpose of the product utilization. A most interesting fact is that the drying process may decrease the share of camphor, while increasing the share of linalool and linalyl acetate which are the most desirable in components in true 
lavender aroma. This result may be a good starting point for considering the improvement of the value of true lavender leaves in comparison to its flowers for flavoring applications.

Supplementary Materials: The following files have been submitted as supplementary materials: mass spectra for unidentified compounds, mentioned in Table 2 Unknown compound mass spectra.pdf.

Author Contributions: Conceptualization, J.Ł. and A.S.; methodology, J.t., A.S., K.J. and M.S.; validation. J.Ł., A.S., K.J., M.S., K.M.; formal analysis. J.Ł., K.J. and M.S.; investigation. J.Ł., K.J. and M.S.; resources. A.S.; data curation. J.Ł.; writing—original draft preparation. J.E. and K.J.; writing—review and editing. J.Ł. and A.S., K.M.; visualization. J.Ł., K.J., M.S. and K.M.; supervision. A.S.; project administration. J.Ł.

Funding: This research was funded by the Faculty of Biotechnology and Food Science, Wrocław University of Environmental and Life Sciences, grant number B030/0003/1 and under the program of the Minister of Science and Higher Education "Strategy of Excellence University of Research" in 2018-2019 project number 0019/SDU/2018/18 in the amount of PLN 700000.

Conflicts of Interest: The authors declare no conflict of interest.

\section{References}

1. Preedy, V.R. Essential Oils in Food Preservation, Flavor and Safety; Elsevier Inc.: London, UK, 2016; ISBN 9780124166417.

2. Prusinowska, R.; Śmigielski, K.B. Composition, biological properties and therapeutic effects of lavender (Lavandula angustifolia L). A review. Herba Pol. 2014, 60, 56-66. [CrossRef]

3. Lesage-Meessen, L.; Bou, M.; Sigoillot, J.C.; Faulds, C.B.; Lomascolo, A. Essential oils and distilled straws of lavender and lavandin: a review of current use and potential application in white biotechnology. Appl. Microbiol. Biotechnol. 2015, 99, 3375-3385. [CrossRef] [PubMed]

4. Ayaz, M.; Sadiq, A.; Junaid, M.; Ullah, F.; Subhan, F.; Ahmed, J. Neuroprotective and anti-aging potentials of essential oils from aromatic and medicinal plants. Front. Aging Neurosci. 2017, 9, 1-16. [CrossRef] [PubMed]

5. Ali, B.; Al-Wabel, N.A.; Shams, S.; Ahamad, A.; Khan, S.A.; Anwar, F. Essential oils used in aromatherapy: A systemic review. Asian Pac. J. Trop. Biomed. 2015, 5, 601-611. [CrossRef]

6. López, V.; Nielsen, B.; Solas, M.; Ramírez, M.J.; Jäger, A.K. Exploring pharmacological mechanisms of lavender (Lavandula angustifolia) essential oil on central nervous system targets. Front. Pharmacol. 2017, 8, 1-8. [CrossRef] [PubMed]

7. Yu, S.H.; Seol, G.H. Lavandula angustifolia Mill. Oil and Its Active Constituent Linalyl Acetate Alleviate Pain and Urinary Residual Sense after Colorectal Cancer Surgery: A Randomised Controlled Trial. Evidence-based Complement. Altern. Med. 2017, 2017, 1-7. [CrossRef] [PubMed]

8. Tomi, K.; Kitao, M.; Murakami, H.; Matsumura, Y.; Hayashi, T. Classification of lavender essential oils: sedative effects of Lavandula oils. J. Essent. Oil Res. 2018, 30, 56-68. [CrossRef]

9. Uritu, C.M.; Mihai, C.T.; Stanciu, G.D.; Dodi, G.; Alexa-Stratulat, T.; Luca, A.; Leon-Constantin, M.M.; Stefanescu, R.; Bild, V.; Melnic, S.; et al. Medicinal plants of the family Lamiaceae in pain therapy: A review. Pain Res. Manag. 2018, 2018, 1-44. [CrossRef]

10. Śmigielski, K.; Prusinowska, R.; Raj, A.; Sikora, M.; Woliñska, K.; Gruska, R. Effect of drying on the composition of essential oil from Lavandula angustifolia. J. Essent. Oil-Bearing Plants 2011, 14, 532-542. [CrossRef]

11. Husnu Can Baser, K.; Buchbauer, G. Handbook of Essential Oils. Science, Technology and Applications; CRC Press: Boca Raton, FL, USA, 2009.

12. Marín, I.; Sayas-Barberá, E.; Viuda-Martos, M.; Navarro, C.; Sendra, E. Chemical Composition, Antioxidant and Antimicrobial Activity of Essential Oils from Organic Fennel, Parsley, and Lavender from Spain. Foods 2016, 5, 18-27. [CrossRef]

13. Hajhashemi, V.; Ghannadi, A.; Sharif, B. Anti-inflammatory and analgesic properties of the leaf extracts and essential oil of Lavandula angustifolia Mill. J. Ethnopharmacol. 2003, 89, 67-71. [CrossRef]

14. Kirimer, N.; Mokhtarzadeh, S.; Demirci, B.; Goger, F.; Khawar, K.M.; Demirci, F. Phytochemical profiling of volatile components of Lavandula angustifolia Miller propagated under in vitro conditions. Ind. Crops Prod. 2017, 96, 120-125. [CrossRef]

15. Mendoza-Poudereux, I.; Kutzner, E.; Huber, C.; Segura, J.; Arrillaga, I.; Eisenreich, W. Dynamics of monoterpene formation in spike lavender plants. Metabolites 2017, 7, 65. [CrossRef] [PubMed] 
16. Rocha, R.P.; Melo, E.C.; Radünz, L.L. Influence of drying process on the quality of medicinal plants: A review. J. Med. Plants Res. 2011, 5, 7076-7084. [CrossRef]

17. Prusinowska, R.; Śmigielski, K. Losses of essential oils and antioxidants during the drying of herbs and spices. A review. Eng. Sci. Technol. 2015, 2, 51-62. [CrossRef]

18. Figiel, A.; Michalska, A. Overall Quality of Fruits and Vegetables Products Affected by the Drying Processes with the Assistance of Vacuum-Microwaves. Int. J. Mol. Sci. 2017, 18, 71. [CrossRef] [PubMed]

19. Figiel, A.; Szumny, A.; Gutiérrez-Ortíz, A.; Carbonell-Barrachina, Á.A. Composition of oregano essential oil (Origanum vulgare) as affected by drying method. J. Food Eng. 2010, 98, 240-247. [CrossRef]

20. Szumny, A.; Figiel, A.; Carbonell-barrachina, A.A. Composition of rosemary essential oil (Rosmarinus officinalis) as affected by drying method. J. Food Eng. 2010, 97, 253-260. [CrossRef]

21. Calín-Sánchez, Á.; Szumny, A.; Figiel, A.; Jałoszyński, K.; Adamski, M.; Carbonell-barrachina, Á.A. Effects of vacuum level and microwave power on rosemary volatile composition during vacuum-microwave drying. J. Food Eng. 2011, 103, 219-227.

22. Sellami, I.H.; Wannes, W.A.; Bettaieb, I.; Berrima, S.; Chahed, T.; Marzouk, B.; Limam, F. Qualitative and quantitative changes in the essential oil of Laurus nobilis L. leaves as affected by different drying methods. Food Chem. 2011, 126, 691-697. [CrossRef]

23. Motevali, A.; Younji, S.; Chayjan, R.A.; Aghilinategh, N.; Banakar, A. Drying kinetics of dill leaves in a convective dryer. Int. Agrophysics 2013, 27, 39-47. [CrossRef]

24. Politowicz, J.; Lech, K.; Sánchez-Rodríguez, L.; Szumny, A.; Carbonell-Barrachina, Á.A. Volatile composition and sensory profile of Cantharellus cibarius Fr. as affected by drying method. J. Sci. Food Agric. 2017, 97, 5223-5232. [CrossRef] [PubMed]

25. Tulek, Y. Drying kinetics of oyster mushroom (Pleurotus ostreatus) in a convective hot air dryer. J. Agric. Sci. Technol. 2011, 13, 655-664.

26. Lech, K.; Figiel, A.; Wojdyło, A.; Korzeniowska, M.; Serowik, M.; Szarycz, M. Drying Kinetics and Bioactivity of Beetroot Slices Pretreated in Concentrated Chokeberry Juice and Dried with Vacuum Microwaves. Dry. Technol. 2015, 33, 1644-1653. [CrossRef]

27. Nöfer, J.; Lech, K.; Figiel, A.; Szumny, A.; Carbonell-Barrachina, Á.A. The Influence of Drying Method on Volatile Composition and Sensory Profile of Boletus edulis. J. Food Qual. 2018, 2018, 1-11. [CrossRef]

28. Figiel, A. Drying kinetics and quality of beetroots dehydrated by combination of convective and vacuum-microwave methods. J. Food Eng. 2010, 98, 461-470. [CrossRef]

29. Calín-Sánchez, Á.; Figiel, A.; Wojdyło, A.; Szarycz, M.; Carbonell-Barrachina, Á.A. Drying of Garlic Slices Using Convective Pre-drying and Vacuum-Microwave Finishing Drying: Kinetics, Energy Consumption, and Quality Studies. Food Bioprocess Technol. 2014, 7, 398-408. [CrossRef]

30. Calín-Sanchez, Á.; Figiel, A.; Szarycz, M.; Lech, K.; Nuncio-Jáuregui, N.; Carbonell-Barrachina, Á.A. Drying Kinetics and Energy Consumption in the Dehydration of Pomegranate (Punica granatum L.) Arils and Rind. Food Bioprocess Technol. 2014, 7, 2071-2083. [CrossRef]

31. Wojdyło, A.; Figiel, A.; Lech, K.; Nowicka, P.; Oszmiański, J. Effect of Convective and Vacuum-Microwave Drying on the Bioactive Compounds, Color, and Antioxidant Capacity of Sour Cherries. Food Bioprocess Technol. 2014, 7, 829-841. [CrossRef]

32. Adams, R.P. Identification of essential oils by ion trap mass spectroscopy; Academic Press: San Diego, CA, USA, 2012.

33. Adaszyńska-Skwirzyńska, M.; Śmist, M.; Swarcewicz, M. Comparison of extraction methods for the determination of essential oil content and composition of lavender leaves. Available online: http:/ / ena.lp. edu.ua:8080/handle/ntb/27086 (accessed on 21 January 2019).

34. Hassanpouraghdam, M.B.; Hassani, A.; Vojodi, L.; Asl, B.H.; Rostami, A. Essential oil constituents of Lavandula ofcinalis Chaix. from Northwest Iran. Chemija 2011, 22, 167-171.

35. Nurzyńska-Wierdak, R.; Zawiślak, G. Chemical composition and antioxidant activity of lavender (Lavandula angustifolia Mill.) aboveground parts. Acta Sci. Pol., Hortorum Cultus 2016, 15, 225-241.

36. An, M.; Haig, T.; Hatfield, P. On-site field sampling and analysis of fragrance from living Lavender (Lavandula angustifolia L.) flowers by solid-phase microextraction coupled to gas chromatography and ion-trap mass spectrometry. J. Chromatogr. A 2001, 917, 245-250. [CrossRef] 
37. Afifi, F.U.; Abu-Dahab, R.; Beltrán, S.; Alcalde, B.B.; Abaza, I.F. GC-MS composition and antiproliferative activity of Lavandula angustifolia Mill. essential oils determined by hydro-distillation, SFE and SPME. Arab. J. Med. Aromat. Plants 2016, 2, 71-85.

38. Fu, J.; Zhao, J.; Zhu, Y.; Tang, J. Rapid Analysis of the Essential Oil Components in Dried Lavender by Magnetic Microsphere-Assisted Microwave Distillation Coupled with HS-SPME Followed by GC-MS. Food Anal. Methods 2017, 10, 2373-2382. [CrossRef]

39. Torabbeigi, M.; Aberoomand Azar, P. Analysis of essential oil compositions of Lavandula angustifolia by HS-SPME and MAHS-SPME followed by GC and GC-MS. Acta Chromatogr. 2013, 25, 571-579. [CrossRef]

40. Mirahmadi, S.F.; Norouzi, R. Influence of Thin Layer Drying on the Essential Oil Content and Composition of Lavandula officinalis. J. Essent. Oil-Bearing Plants 2016, 19, 1537-1546. [CrossRef]

41. Milojevic, S.; Radosavljevic, D.; Pavicevic, V.; Pejanovic, S.; Veljkovic, V. Modeling the kinetics of essential oil hydrodistillation from plant materials. Hem. Ind. 2013, 67, 843-859. [CrossRef]

42. Baydar, H.; Erbaş, S. Effects of harvest time and drying on essential oil properties in lavandin (Lavandula $\times$ intermedia Emeric ex Loisel.). Acta Hortic. 2009, 826, 377-382. [CrossRef]

43. Ghasemi, A.; Salehi, S.; Craker, L. Effect of drying methods on qualitative and quantitative properties of essential oil from the aerial parts of coriander. J. Dermatol. Sci. 2017, 4, 35-40.

44. Kim, N.S.; Lee, D.S. Comparison of different extraction methods for the analysis of fragrances from Lavandula species by gas chromatography-mass spectrometry. J. Chromatogr. A 2002, 982, 31-47. [CrossRef]

45. Da Porto, C.; Decorti, D. Analysis of the volatile compounds of flowers and essential oils from Lavandula angustifolia cultivated in northeastern Italy by headspace solid-phase microextraction coupled to gas chromatography-mass spectrometry. Planta Med. 2008, 74, 182-187. [CrossRef] [PubMed]

46. Aghbashlo, M.; kianmehr, M.H.; Samimi-Akhijahani, H. Influence of drying conditions on the effective moisture diffusivity, energy of activation and energy consumption during the thin-layer drying of berberis fruit (Berberidaceae). Energy Convers. Manag. 2008, 49, 2865-2871. [CrossRef]

47. Alibas, I. Characteristics of chard leaves during microwave, convective, and combined microwave-convective drying. Dry. Technol. 2006, 24, 1425-1435. [CrossRef]

48. Dadali, G.; Apar, D.K.; Özbek, B. Microwave drying kinetics of okra. Dry. Technol. 2007, 25, 917-924. [CrossRef]

Sample Availability: Samples of the compounds are not available from the authors.

(C) 2019 by the authors. Licensee MDPI, Basel, Switzerland. This article is an open access article distributed under the terms and conditions of the Creative Commons Attribution (CC BY) license (http://creativecommons.org/licenses/by/4.0/). 